\title{
Complexity of the Stable Model Semantics for Queries on Incomplete Databases
}

\author{
Jos de Bruijn $^{1}$ and Stijn Heymans ${ }^{2}$ \\ 1 KRDB Research Center, Free University of Bozen-Bolzano, Italy \\ debruijneinf.unibz.it \\ 2 Institute of Information Systems, Vienna University of Technology, Austria \\ heymansekr.tuwien.ac.at
}

\begin{abstract}
We study the complexity of consistency checking and query answering on incomplete databases for languages ranging from non-recursive Datalog to disjunctive Datalog with negation under the stable model semantics. We consider both possible and certain answers and both closed- and open-world interpretation of $C$-databases with and without conditions. By reduction to stable models of logic programs we find that, under closed-world interpretation, adding negation to (disjunctive) Datalog does not increase the complexity of the considered problems for C-databases, but certain answers for databases without conditions are easier for Datalog without than with negation. Under open-world interpretation, adding negation to non-recursive Datalog already leads to undecidability, but the complexity of certain answers for negation-free queries is the same as under closed-world interpretation.
\end{abstract}

\section{Introduction}

In applications of relational databases a need often arises for representing incomplete information [5], typically in the form of null values. For example, in data exchange $[8,17]$ anomalies in the semantics for solutions may arise if nulls are not treated with care. In data integration $[1,12,16]$ incomplete information arises when integrating different complete data sources using a global schema; a materialized view may be incomplete with respect to local sources and local sources may be incomplete with respect to the global schema or constraints of other sources.

Null values in incomplete databases are represented using variables. A single database represents several possible instances, called representations. In our treatment we follow the landmark paper by Imieliński and Lipski [14], which considers both closedworld and open-world interpretation of incomplete databases. In the former, representations are in direct correspondence with valuations of the variables; each tuple in a representation is the valuation of a tuple in the database. In the latter, representations may include additional tuples not originating from the database. In addition, local conditions may be attached to tuples and a global condition to the database. Such incomplete databases with conditions are called $C$-databases.

So far, most research on query answering has been concerned with first-order and Datalog queries [25,3], and has focused mainly on data complexity. However, since those landmark papers, the formal properties of more expressive query languages such 
as Datalog with disjunction [7] and (unstratified) negation with the accompanying Stable Model Semantics [9] (Datalog ${ }^{\neg} \vee$ ) established themselves firmly as well-accepted expressive Knowledge Representation languages. Sufficient reason for having a closer look again at those query languages for incomplete databases and thus going beyond PTime queries - queries that can be answered in polynomial time on complete databases (e.g., stratified Datalog [2]).

We study the data and combined complexity of consistency, and possible and certain answers for languages ranging from nonrecursive Datalog to Datalog ${ }^{\neg, \vee}$. We consider both the open- and closed-world interpretation of C-databases with and without conditions. Our main contributions are summarized as follows:

- We show that answering Datalog ${ }^{\neg} \vee$ queries on incomplete databases under closedworld interpretation can be reduced to common reasoning tasks in logic programming, by an encoding of incomplete databases into logic programs.

- We present complete pictures of the data and combined complexity of consistency, and possible and certain answers for languages ranging from non-recursive Datalog to Datalog $\urcorner, \vee$, complementing earlier results $[3,25]$ with combined complexity results for (fragments of) stratified Datalog $\urcorner$ queries and novel data and combined complexity results for queries beyond PTime. The results for closed-world interpretation are summarized in Table 1 on page 10, and for open-world interpretation in Table 2 on page 11. - Finally, we show that results about checking uniform and strong equivalence of queries from the areas of complete databases and logic programming apply immediately to the case of incomplete databases.

To the best of our knowledge, ours are the first results about answering Datalog queries with disjunction and/or stable model negation on incomplete databases. Related to Datalog $\urcorner$ queries on incomplete databases are the techniques for consistent query answering in data integration based under the local-as-view using Datalog $\urcorner$ programs, by Bertossi and Bravo [4]. The precise relationship with query answering on incomplete databases is an open question.

Under open-world interpretation, adding negation leads to undecidability of consistency and query answering, by undecidability of finite satisfiability in first-order logic [22]. Under closed-world interpretation, for all query languages ranging from nonrecursive Datalog to Datalog $\urcorner$, the data complexity of possible answers on databases without conditions is NP-complete and of certain answers on C-databases it is coNPcomplete; for certain answers coNP-completeness holds additionally for Datalog $\vee$ queries. For positive queries these results also apply under open-world interpretation. This shows that, for possible answers and for certain answers on general C-databases, there is no computational justification for restricting oneself to PTime languages such as Datalog and stratified Datalog $\urcorner$.

In Section 2 we review incomplete databases and define Datalog $\neg, \vee$ queries. In Section 3 we reduce query answering under closed-world interpretation to standard logic programming reasoning tasks. We present our complexity analysis in Section 4 . Finally, we discuss related and future work in Sections 5 and 6.

An extended version of this paper, which includes the proofs of Propositions 4, 7, 10, and 11 , can be found online at:

http://www.debruijn.net/publications/sm-incomplete-db.pdf. 


\section{Incomplete Databases and Queries}

We consider C-databases, as defined by Imieliński and Lipski [14], and the Stable Model Semantics for logic programs, as defined by Gelfond and Lifschitz [9].

Incomplete Databases Let $\mathcal{D}$ be a countably infinite set of constants, called the domain, and let $\mathcal{V}$ be a finite set of variables, disjoint from $\mathcal{D}$. A condition $\psi$ is a formula of the form $\varphi_{1} \vee \cdots \vee \varphi_{m}$, where $\varphi_{j}$ are conjunctions of equality atoms $x=y$ and inequality atoms $x \neq y$, with $x, y \in \mathcal{D} \cup \mathcal{V}$. A $C$-table (Conditional table) of arity $n$ is a finite subset of $(\mathcal{D} \cup \mathcal{V})^{n}$ such that a local condition $\phi_{t}$ is associated with each tuple $t$ in the relation. We sometimes omit $\phi_{t}$ if it is $x=x$.

A schema is a list $\mathcal{T}=R_{1}, \ldots, R_{k}$ of predicate symbols $R_{i}$ each with an arity $n_{i} \geq$ 1. We assume a constant bound $l$ on the arities. A $C$-database over $\mathcal{T}$ is a tuple $\mathbf{T}=$ $\left(T_{1}, \ldots, T_{k}\right)$ with associated condition $\Phi_{\mathbf{T}}$, such that each $T_{i}$ is a C-table with arity $n_{i}$. We write individual tuples $\left(a_{1}, \ldots, a_{n_{i}}\right) \in T_{i}$ as $R_{i}\left(a_{1}, \ldots, a_{n_{i}}\right)$; if $R_{i}\left(a_{1}, \ldots, a_{n_{i}}\right)$ contains no variables, it is a fact. With preds $(\mathbf{T})$ we denote the set $\left\{R_{1}, \ldots, R_{k}\right\}$. We call a C-database condition-free if every condition is $x=x$. A complete database or instance $I$ is a variable- and condition-free $\mathrm{C}$-database.

Validity of variable-free conditions is defined as follows: $c_{1}=c_{1}$ is valid; $c_{1} \neq c_{2}$ is valid, for $c_{1}, c_{2}$ distinct constants; this extends to conditions in the natural way. A valuation is a mapping $\sigma: \mathcal{V} \cup \mathcal{D} \rightarrow \mathcal{D}$ such that $\sigma(c)=c$, for every $c \in \mathcal{D}$. This extends to tuples and conditions in the natural way. For C-tables $T$ and $\mathbf{C}$-databases $\mathbf{T}$ we define $\sigma(T)=\left\{\sigma(t) \mid t \in T \& \sigma\left(\phi_{t}\right)\right.$ is valid $\}$ and $\sigma(\mathbf{T})=\left(\sigma\left(T_{1}\right), \ldots, \sigma\left(T_{k}\right)\right)$.

The closed-world interpretation (CWI) of a C-database $\mathbf{T}$ with arity $\left(n_{1}, \ldots, n_{k}\right)$ is defined as:

$$
\operatorname{rep}(\mathbf{T})=\left\{\sigma(\mathbf{T}) \mid \sigma \text { is a valuation such that } \sigma\left(\Phi_{\mathbf{T}}\right) \text { is valid }\right\}
$$

The open-world interpretation (OWI) of $\mathbf{T}$ is defined as:

$$
\begin{aligned}
\operatorname{Rep}(\mathbf{T})=\left\{\mathbf{R} \subseteq \mathcal{D}^{n_{1}} \times \cdots \times \mathcal{D}^{n_{k}} \mid \exists \sigma . \sigma\left(\Phi_{\mathbf{T}}\right)\right. \text { is valid, } \\
\qquad \sigma(\mathbf{T}) \subseteq \mathbf{R}, \text { and } \mathbf{R} \text { is finite }\}
\end{aligned}
$$

Lemma 1 (Implicit in [14]). Let $\mathbf{T}$ be a $C$-database. Then, rep $(\mathbf{T}) \subseteq R e p(\mathbf{T})$ and for every $I \in \operatorname{Rep}(\mathbf{T})$ there is an $I^{\prime} \in \operatorname{rep}(\mathbf{T})$ such that $I^{\prime} \subseteq I$.

Datalog ${ }^{\neg, \vee}$ Queries Atoms are of the form $p\left(a_{1}, \ldots, a_{n}\right)$, where the $a_{i}$ 's are terms and $p$ is an $n$-ary predicate symbol, $n \geq 1$. Positive literals are atoms $\alpha$ and negative literals are negated atoms not $\alpha$. A Datalog ${ }^{\neg, \vee}$ rule $r$ is of the form:

$$
h_{1} \vee \cdots \vee h_{l} \leftarrow b_{1}, \ldots, b_{k}
$$

where the $h_{i}$ 's are atoms and the $b_{j}$ 's are literals, such that every variable in $r$ occurs in some positive $b_{j}$. We call $H(r)=\left\{h_{1}, \ldots, h_{l}\right\}$ the head and $B(r)=\left\{b_{1}, \ldots, b_{k}\right\}$ the body of $r$. If $r$ contains no negation, then it is a Datalog ${ }^{\vee}$ rule. If $l=1$, then $r$ is a Datalog $\urcorner$ rule. If $r$ is both a Datalog $\vee$ and Datalog $\urcorner$ rule, then it is a Datalog rule. 
A Datalog $\urcorner, \vee$ program $P$ is a countable set of Datalog $\urcorner, \vee$ rules. Datalog $\urcorner$, Datalog ${ }^{\vee}$, and Datalog programs are defined analogously.

The set of predicate symbols of $P$, denoted $\operatorname{preds}(P)$, is partitioned into sets of $i n$ tentional $(\operatorname{int}(P))$ and extensional $(\operatorname{ext}(P))$ predicates such that there is no $p \in \operatorname{ext}(P)$ in the head of any $r \in P$. We assume that each variable occurs in at most one $r \in P$.

The dependency graph of $P$ is a directed graph $G(P)=\langle N, E\rangle: N=\operatorname{preds}(P)$ and $E$ is the smallest set that includes an edge $(p, q) \in \operatorname{preds}(P)^{2}$ if there is an $r \in P$ such that $p$ in some $h \in H(r)$ and $q$ in some $b \in B(r) ;(p, q)$ is labeled negative if $b$ is a a negative literal. $P$ is non-recursive if $G(P)$ contains no cycles and stratified if $G(P)$ contains no cycles involving a negative edge. We use the prefixes nr- and st- for class of non-recursive and stratified programs.

Given a set $\Delta \subseteq \mathcal{D}$, the grounding of $P$ with respect to $\Delta$, denoted $\operatorname{gr}_{\Delta}(P)$, is defined as the union of all substitutions of variables in $P$ with elements of $\Delta$.

Definition 1 (Queries). If $\mathrm{X}$ is a class of programs, then an $\mathrm{X}$ query $Q$ with signature $\left(e_{1}, \ldots, e_{n}\right) \rightarrow\left(o_{1}, \ldots, o_{m}\right)$ is a finite $\mathrm{X}$ program without constants and without empty rule heads such that $\left\{e_{1}, \ldots, e_{n}\right\}=\operatorname{ext}(Q)$ are the input and $\left\{o_{1}, \ldots, o_{m}\right\} \subseteq \operatorname{int}(Q)$ are the output predicates. $Q$ is well-defined with respect to a database $\mathbf{T}$ if ext $(Q) \subseteq$ preds $(\mathbf{T})$.

We assume in the remainder that all queries are well-defined with respect to the database under consideration; further, let $\Delta$ be a set of constants, $P$ a program, $I$ an instance, and $\mathbf{I}$ a set of instances.

An interpretation $M$ is a set of facts formed using predicate symbols in $\operatorname{preds}(P)$ and constants $\Delta$. Given a set of predicate symbols or constants $\Upsilon$, with $\left.M\right|_{\Upsilon}$ we denote the restriction of $M$ to $\Upsilon$.

If $P$ is negation- and variable-free, $M$ is a model of $P$ if, for every $r \in P$, whenever $B(r) \subseteq M, H(r) \cap M \neq \emptyset$. The reduct $P^{M, \Delta}$ is obtained from $g r_{\Delta}(P)$ by (a) removing every rule $r \in g r_{\Delta}(P)$ such that not $b \in B(r)$ for some $b \in M$ and (b) removing all negative literals from the remaining rules.

$M$ is a stable $\Delta$-model of $P$ with respect to $I$ if $\left.M\right|_{\text {ext }(P)}=\left.I\right|_{\text {ext }(P)}, M$ is a model of $P^{M, \Delta}$ and there is no model $M^{\prime}$ of $P^{M, \Delta}$ such that $\left.M^{\prime}\right|_{\operatorname{ext}(P)}=\left.M\right|_{\operatorname{ext}(P)}$ and $M^{\prime} \varsubsetneqq M$. We leave out $I$ if $I=\emptyset$ and $\Delta$ if $\Delta=\mathcal{D}$. We note that if $\Delta$ includes the constants in $I$ and $P$, then the stable $\Delta$-models of $P$ with respect to $I$ are the same as the stable models of $P \cup I$.

Example 1. Let $\Delta=\{a\}$. Consider the instance $I=\{p(a)\}$ and the program $P=$ $\{q(x) \vee r(x) \leftarrow p(x)$, not $s(x)\} . M=\{p(a), q(a)\}$ is a model of the reduct $P^{M, \Delta}=$ $\{q(a) \vee r(a) \leftarrow p(a)\}$ and a stable $\Delta$-model of $P$ with respect to $I$; the other stable $\Delta$-model is $\{p(a), r(a)\}$.

Definition 2 (Query Answers). Let I be an instance, I a set of instances, and $Q$ a Datalog ${ }^{\neg, \vee}$ query with signature $\left(e_{1}, \ldots, e_{n}\right) \rightarrow\left(o_{1}, \ldots, o_{m}\right)$.

$$
\begin{aligned}
& Q(I)=\left\{\left(\left.M\right|_{\left\{o_{1}\right\}}, \ldots,\left.M\right|_{\left\{o_{m}\right\}}\right) \mid M \text { is a stable model of } Q \text { with respect to } I\right\} \\
& Q(\mathbf{I})=\bigcup\{Q(I) \mid I \in \mathbf{I}\}
\end{aligned}
$$

The closed-world interpretation of a query $Q$ on a C-database $\mathbf{T}$ is written $Q(\operatorname{rep}(\mathbf{T}))$ and the open-world interpretation is written $Q(\operatorname{Rep}(\mathbf{T}))$. 


\section{Logic Programming Characterization of Queries under CWI}

We reduce queries on incomplete databases under closed-world interpretation to logic programs with negation. Specifically, we show that there is a polynomial embedding of C-databases $\mathbf{T}$ into Datalog $\urcorner$ programs $P_{\mathbf{T}}$ such that the answers to a query $Q$ on T correspond with the stable models of $Q \cup P_{\mathbf{T}}$ with respect to the output predicates $\left(o_{1}, \ldots, o_{m}\right)$.

Recall that the domain $\mathcal{D}$ is infinite, and thus there may be infinitely many valuations for a given variable in $\mathbf{T}$. The following lemma shows we need to consider only a finite subset.

Lemma 2 (Implicit in [3]). Let $Q$ be a Datalog $^{\neg, \vee}$ query, $\mathbf{T}$ a C-database, $\Delta \subset \mathcal{D}$ include the constants in $\mathbf{T}$, and $V$ the set of variables in $\mathbf{T}$. Then there is a set of constants $\Delta^{\prime} \subset \mathcal{D}$ with cardinality $|V|$ such that $\Delta \cap \Delta^{\prime}=\emptyset$ and.

$$
\left.Q(\operatorname{rep}(\mathbf{T}))\right|_{\Delta}=\left\{\left.I\right|_{\Delta} \mid \sigma: V \rightarrow \Delta \cup \Delta^{\prime}, I \in Q(\sigma(\mathbf{T})), \text { and } \sigma\left(\Phi_{\mathbf{T}}\right) \text { is valid }\right\}
$$

Note that for given $Q, \mathbf{T}, \Delta$, and $V$, such a $\Delta^{\prime}$ is finite, since $|V|$ is finite.

Definition 3. Let $\mathbf{T}$ be a $C$-database and $\Delta \subset \mathcal{D}$ include constants in $\mathbf{T}$. For each tuple $t=R(\boldsymbol{a})$ in $\mathbf{T}$, with $\phi_{t}=\varphi_{t, 1} \vee \cdots \vee \varphi_{t, m}$, the program $P_{\mathbf{T}, \Delta}$ contains the rules

$$
R(\boldsymbol{a}) \leftarrow \varphi_{t, i}^{\prime}, v_{x_{1}}\left(x_{1}\right), \ldots, v_{x_{k}}\left(x_{k}\right)
$$

for $1 \leq i \leq m$, where $\varphi_{t, i}^{\prime}$ is obtained from $\varphi_{t, i}$ by replacing ' $\wedge$ ' with ',', and $x_{1}, \ldots, x_{k}$ are the variables occurring in $t$ or $\varphi_{t, i}$.

$P_{\mathbf{T}, \Delta}$ contains $D(c) \leftarrow$, for every $c \in \Delta \cup \Delta^{\prime}$, with $\Delta^{\prime}$ as in Lemma 2,

$$
\begin{array}{rlrl}
v_{x}(z) \leftarrow \operatorname{not} v_{x}^{\prime}(z), D(z) & \leftarrow v_{x}(z), v_{x}(y), z \neq y \\
v_{x}^{\prime}(z) \leftarrow \operatorname{not} v_{x}(z), D(z) & e_{x} & \leftarrow v_{x}(z) \\
& \leftarrow \operatorname{not} e_{x}
\end{array}
$$

for every variable $x$ in $\mathbf{T}$. Finally, for $\Phi_{\mathbf{T}}=\varphi_{\mathbf{T}, 1} \vee \cdots \vee \varphi_{\mathbf{T}, l}, P_{\mathbf{T}, \Delta}$ contains

$$
g \leftarrow \varphi_{\mathbf{T}, i}^{\prime}, v_{x_{1}}\left(x_{1}\right), \ldots, v_{x_{k}}\left(x_{k}\right) \quad \leftarrow \operatorname{not} g
$$

for $1 \leq i \leq l$, where $\varphi_{\mathbf{T}, i}^{\prime}$ is obtained from $\varphi_{\mathbf{T}, i}$ as before and $x_{1}, \ldots, x_{k}$ are the variables in $\varphi_{\mathbf{T}, i} . P_{\mathbf{T}, \Delta}$ contains no other rules.

Note that equality and inequality can be straightforwardly axiomatized using Datalog $\urcorner$ rules, such that $P_{\mathbf{T}, \Delta}$ is indeed a Datalog $\urcorner$ program.

Intuitively, the rules (5) ensure the presence of an atom $v_{x}(c)$ in every stable model, indicating that the variable $x$ is assigned to $c$. The constraints ensure that there is such a guess for each variable and this guess is unique. The rules (4) subsequently ensure evaluating the conditions.

The following proposition establishes correspondence between the answers to $Q$ on $\operatorname{rep}(\mathbf{T})$ and the stable models of $Q \cup P_{\mathbf{T}, \Delta}$. 
Proposition 1. Let $Q$ be a query with signature $\left(e_{1}, \ldots, e_{n}\right) \rightarrow\left(o_{1}, \ldots, o_{m}\right)$ on a $C$-database $\mathbf{T}$ and let $\Delta$ be a superset of the set of constants in $\mathbf{T}$. Then,

$$
\left.Q(\operatorname{rep}(\mathbf{T}))\right|_{\Delta}=\left.\left\{\left(\left.M\right|_{\left\{o_{1}\right\}}, \ldots,\left.M\right|_{\left\{o_{m}\right\}}\right) \mid M \text { is a stable model of } Q \cup P_{\mathbf{T}, \Delta}\right\}\right|_{\Delta}
$$

Proof. One can verify that $M$ is a stable model of $P_{\mathbf{T}, \Delta}$ iff $M=\sigma(\mathbf{T})$ for a $\sigma: V \rightarrow$ $\Delta \cup \Delta^{\prime}$ such that $\sigma\left(\Phi_{\mathbf{T}}\right)$ is valid. The proposition then straightforwardly follows from the definition and Lemma 2.

Observe that the grounding of the program $P_{\mathbf{T}, \Delta}$ is in general exponential in the size of $\mathbf{T}, \Delta$, since the size of the non-ground rules (4) depends on the size of $\mathbf{T}$. However, we will see in Proposition 5 that using an intelligent polynomial grounding, the stable models of $P_{\mathbf{T}, \Delta}$ can be computed in time NP.

Example 2. Consider a C-database $\mathbf{T}$ with ternary table $T$ describing the flights of a plane on a particular day. $\mathbf{T}$ contains the tuples $t_{1}=T\left(v, x_{1}, y_{1}\right)$ and $t_{2}=T\left(i, x_{2}, y_{2}\right)$, with variables $x_{1}, x_{2}, y_{1}, y_{2}$, indicating that the plane flies from $v$ to a destination $x_{1}$ with a pilot $y_{1}$ and from $i$ to $x_{2}$ with $y_{2}$. As $m g$ and $m c$ are the only pilots certified to fly on $i, t_{1}$ has associated condition $x_{1} \neq i \vee y_{1}=m g \vee y_{1}=m c$ and $t_{2}$ has condition $y_{2}=m g \vee y_{2}=m c$. Additionally, a pilot may not fly two stretches, hence the global condition $y_{1} \neq y_{2} \wedge x_{1} \neq v \wedge x_{2} \neq i$.

Let $\Delta$ be the set of constants in $\mathbf{T}$. Besides the guess rules (5), $P_{\mathbf{T}, \Delta}$ contains

$$
\begin{aligned}
& T\left(v, x_{1}, y_{1}\right) \leftarrow x_{1} \neq i, v_{x_{1}}\left(x_{1}\right), v_{y_{1}}\left(y_{1}\right) \quad T\left(i, x_{2}, y_{2}\right) \leftarrow y_{2}=m g, v_{x_{2}}\left(x_{2}\right), v_{y_{2}}\left(y_{2}\right) \\
& T\left(v, x_{1}, y_{1}\right) \leftarrow y_{1}=m g, v_{x_{1}}\left(x_{1}\right), v_{y_{1}}\left(y_{1}\right) T\left(i, x_{2}, y_{2}\right) \leftarrow y_{2}=m c, v_{x_{2}}\left(x_{2}\right), v_{y_{2}}\left(y_{2}\right) \\
& T\left(v, x_{1}, y_{1}\right) \leftarrow y_{1}=m c, v_{x_{1}}\left(x_{1}\right), v_{y_{1}}\left(y_{1}\right) \\
& \quad g \leftarrow y_{1} \neq y_{2}, x_{1} \neq v, x_{2} \neq i, v_{y_{1}}\left(y_{1}\right), v_{y_{2}}\left(y_{2}\right), v_{x_{1}}\left(x_{1}\right), v_{x_{2}}\left(x_{2}\right) \quad \leftarrow \text { not } g
\end{aligned}
$$

Among the stable models of $P_{\mathbf{T}, \Delta}$ (restricted to $\operatorname{preds}(\mathbf{T})$ ) are $M_{1}=\{T(v, i, m g$ ), $T(i, v, m c)\}$ and $M_{2}=\{T(v, i, m c), T(i, v, m g)\}$. One can verify that these indeed correspond to elements of $\operatorname{rep}(\mathbf{T})$. Consider the Datalog $\urcorner$ query $Q$

$$
\begin{aligned}
\operatorname{flying}(x, y, z) & \leftarrow T(x, y, z) \\
\text { flying }(x, y, z) & \leftarrow T(x, u, z), \operatorname{flying}(u, y, z) \\
\text { roundtrip }(z) & \leftarrow \operatorname{flying}(x, x, z) \\
\operatorname{stranded}(x) & \leftarrow \operatorname{not} \text { roundtrip }(z), \operatorname{flying}(x, y, z)
\end{aligned}
$$

where flying is the transitive closure of the trips in $T$ and the pilot is stranded if the departure and final destination do not coincide. The output predicate is stranded.

Consider the stable models $M_{1}^{\prime}$ and $M_{2}^{\prime}$ of $Q \cup P_{\mathbf{T}, \Delta}$, which are extensions of $M_{1}$ and $M_{2}$, respectively. Both $M_{1}^{\prime}$ and $M_{2}^{\prime}$ contain stranded $(m g)$ and stranded $(m c)$. However, $Q \cup P_{\mathbf{T}, \Delta}$ also has the stable models $\left\{T\left(v, c_{x_{1}}, c_{y_{1}}\right), T(i, v, m c)\right.$, stranded $(m c)$, $\left.\operatorname{stranded}\left(c_{y_{1}}\right)\right\}$ and $\left\{T\left(v, c_{x_{1}}, c_{y_{1}}\right), T(i, v, m g)\right.$, stranded $\left.(m g), \operatorname{stranded}\left(c_{y_{1}}\right)\right\}$, and so neither stranded $(m g)$ nor stranded $(m c)$ is included in every stable model.

\section{Complexity Analysis}

In this section we study the complexity of checking consistency (cons) and of query answering, under the possible (poss) and certain (cert) answer semantics. 
We consider two notions of complexity (cf. [23]): combined complexity is measured in the combined size of the database and the query and data complexity is measured in the size of the database - the query is considered fixed. We consider the following decision problems. As inputs (in parentheses) we consider a set of facts $A$, a C-database $\mathbf{T}$, and a query $Q$.

$\operatorname{cons}(\mathbf{T}, Q) \quad$ question: is there an $I \in Q(\operatorname{rep}(\mathbf{T}))$ such that $I \neq \emptyset$ ?

$\operatorname{poss}(A, \mathbf{T}, Q) \quad$ question: is there an $I \in Q(\operatorname{rep}(\mathbf{T}))$ such that $A \subseteq I$ ?

$\operatorname{cert}(A, \mathbf{T}, Q) \quad$ question: for all $I \in Q(\operatorname{rep}(\mathbf{T})), A \subseteq I$ ?

cons $^{Q}$, poss ${ }^{Q}$, and $\operatorname{cert}^{Q}$ are like the above except that $Q$ is not part of the input.

We denote the consistency and certain answer problems under open-world interpretation with the symbols Cons and Cert, respectively. Their definitions are obtained from the above by replacing $\operatorname{rep}(\cdot)$ with $\operatorname{Rep}(\cdot)$. We do not consider possible answers in the open-world case, since representations may include facts not justified by tuples in the database.

With a problem $Y$ (resp., $Y^{Q}$ ) for a class $X$ of queries, we mean the restriction of the problem $Y$ (resp., $Y^{Q}$ ) such the queries $Q$ in the input (resp., parameter) are in the class $X$. We use the following notation for complexity classes: LSpace (logarithmic space), PTime, NP, coNP, $\Sigma_{2}^{p}=\mathrm{NP}^{\mathrm{NP}}, \Pi_{2}^{p}=$ coNP $^{\mathrm{NP}}$, PSpace, Exp (exponential time), NExp, coNExp, NExp ${ }^{N P}$, and coNExp ${ }^{N P}$. See, e.g., [6, Section 3] for definitions.

We consider the closed-world interpretation in Section 4.1 and the open-world interpretation in Section 4.2.

\subsection{Complexity of Closed-World Interpretation}

In order to give a full picture of the complexity, we repeat some results from literature of query answering over incomplete databases in Proposition 2 and 3. Queries in these propositions have no negation or only stratified negation such that a stable model semantics coincides with the usual minimal model semantics as defined in the respective literature. The following result is due to Abiteboul et al. [3].

Proposition 2 ([3]). The problem poss ${ }^{Q}$ is NP-complete and cert ${ }^{Q}$ is coNP-complete for $\mathrm{nr}$-Datalog, nr-Datalog $\urcorner$, Datalog, and st-Datalog $\urcorner$ queries.

In addition, Grahne [11] showed that when restricting local conditions $\phi_{t}$ to conjunctions of equalities and the global condition $\Phi_{\mathbf{T}}$ to a conjunction of Horn clauses, the problem $\operatorname{cert}^{Q}$ can be solved in PTime for Datalog queries.

The hardness results in the following proposition follow from the hardness results for the case of complete databases; see [6,7,26]. Observe that the complement of poss for Datalog $\vee$ queries is easily reduced to cert for stratified Datalog ${ }^{\urcorner, \vee}$ queries; $\Pi_{2}^{p}$ (resp., $\operatorname{coNExp}^{\mathrm{NP}}$ )-hardness of $\operatorname{cert}^{Q}$ (resp., cert) for st-Datalog ${ }^{\neg, \vee}$ follows immediately from $\Sigma_{2}^{p}$ (resp., $\mathrm{NExp}^{\mathrm{NP}}$ )-hardness of poss ${ }^{Q}$ (resp., poss) for Datalog ${ }^{\vee}$ queries [7]. Observe also that the problems poss and cert correspond for nr-Datalog queries on complete databases; PSpace-hardness of cert was established by Vorobyov and Voronkov [26]. 
Proposition 3. The problem

- poss $^{Q}$ is $\Sigma_{2}^{p}$-hard for Datalog $\vee$ queries,

- cert $^{Q}$ is $\Pi_{2}^{p}$-hard for st-Datalog $\neg, \vee$ queries,

- poss and cert are PSpace-hard for nr-Datalog queries,

- poss is NExp ${ }^{N P}$-hard for Datalog ${ }^{\vee}$ queries, and

- cert is coNExp ${ }^{N P}$-hard for $\mathrm{st}^{-D a t a l o g}{ }^{\neg, \vee}$ queries.

We state our novel hardness result of combined complexity for Datalog in Proposition 4. Our novel membership results for queries beyond Datalog are in Propositions 5 and 6 . The results are summarized in Table 1 on page 10.

Proposition 4. The problem poss is NExp-hard and cert is coNExp-hard for Datalog queries.

Proof (Sketch). The proof is by an encoding of nondeterministic Turing machines that run in exponential time into Datalog queries $Q$ on C-databases T. $Q$, which has one output predicate accept, encodes all possible transitions of the machine, using binary coding for time points and positions on the tape. $\mathbf{T}$ encodes a guess for each time point j. Conditions ensure that in a given representation, exactly one guess is made for each time point. We have that $\operatorname{accept}(1) \in I$ for some (resp., all) $I \in Q(\operatorname{rep}(\mathbf{T}))$ iff some (resp., all) run(s) of $T$ are accepting. Consequently, poss is NExp-hard and cert is coNExp-hard for Datalog queries.

The complete encoding can be found in the extended version.

We obtain the following membership results with the help of the reduction to logic programs in Section 3 (see Proposition 1).

Proposition 5. The problem

- poss ${ }^{Q}$ is in NP and cert ${ }^{Q}$ is in coNP for Datalog $\urcorner$ queries,

- poss $^{Q}$ is in $\Sigma_{2}^{p}$ and cert ${ }^{Q}$ is in $\Pi_{2}^{p}$ for Datalog ${ }^{\urcorner, \vee}$ queries,

- poss and cert are in PSpace for $\mathrm{nr}-$ Datalog $\urcorner$ queries,

- poss is in NExp and cert is in coNExp for Datalog $\urcorner$ queries, and

- poss is in $N \operatorname{Exp}^{N P}$ and cert is in coNExp ${ }^{N P}$ for Datalog ${ }^{\urcorner, \vee}$ queries.

Proof. Let $\mathbf{T}$ be a C-database, $A$ a set of facts, $Q$ a Datalog ${ }^{\neg, \vee}$ query, $\Delta$ the set of constants occurring in $\mathbf{T}$ or $A, V$ the set of variables in $\mathbf{T}$, and $P_{\mathbf{T}, \Delta}$ the logic program that encodes $\mathbf{T}$ (see Definition 3 ). Without loss of generality we assume that the facts in $A$ all involve output predicates of $Q$. By Proposition 1, $\operatorname{poss}(A, \mathbf{T}, Q)$ (resp., $\operatorname{cert}(A, \mathbf{T}, Q))$ iff for some (resp, all) stable model(s) $M$ of $P_{\mathbf{T}, \Delta} \cup Q, A \subseteq M$.

Consider the following algorithm for computing the stable models of $P_{\mathbf{T}, \Delta} \cup Q$. Observe that for each stable model $M$ of $P_{\mathbf{T}, \Delta} \cup Q$ it must hold, by the rules (5), that (†) for each $v_{x_{i}}$, with $x_{i} \in V$, there is exactly one $v_{x_{i}}\left(t_{x_{i}}\right) \in M$.

1. Guess an interpretation $M$ for $P_{\mathbf{T}, \Delta} \cup Q$ such that (†) holds.

2. Check whether $M$ is a minimal model of $\left(g r_{\Delta \cup \Delta^{\prime}}\left(P_{\mathbf{T}, \Delta}^{\prime} \cup Q\right)\right)^{M}$, where $P_{\mathbf{T}, \Delta}^{\prime}$ is obtained from $P_{\mathbf{T}, \Delta}$ by replacing every $v_{x_{i}}\left(x_{i}\right)$ with $v_{x_{i}}\left(t_{x_{i}}\right)$. 
The size of the guess $M$ is clearly polynomial in $\mathbf{T}$. The reduct $\left(g r_{\Delta \cup \Delta^{\prime}}\left(P_{\mathbf{T}, \Delta}^{\prime} \cup Q\right)\right)^{M}$ can be computed in time polynomial in the size of $\mathbf{T}$ (since every predicate has bounded arity) and exponential in the combined size of $\mathbf{T}$ and $Q$. Then, checking whether $M$ is a minimal model of the reduct can be done in PTime if $Q \in$ Datalog $\urcorner$ and with an NP oracle if $Q \in$ Datalog $^{\urcorner, \vee}$ (cf. [6]). The first, second, fourth, and fifth bullet follow immediately.

Finally, if $Q$ does not contain recursion, it is not necessary to consider the complete grounding; the algorithm can consider the possible variable substitutions one at a time. This requires polynomial space; the third bullet follows from the fact that nondeterministic PSpace $=$ PSpace.

For determining the complexity of the certain answer semantics for Datalog ${ }^{\vee}$ queries we exploit the fact that entailment from Datalog ${ }^{\vee}$ programs corresponds to propositional consequence from its ground instantiation.

Proposition 6. The problem cert ${ }^{Q}$ is in coNP and the problem cert is in coNExp for Datalog $\vee$ queries.

Proof. We have that $\operatorname{cert}^{Q}(A, \mathbf{T})$ iff $A \subseteq M$ for every $I \in \operatorname{rep}(\mathbf{T})$ and stable model $M$ of $Q \cup I$, which is in turn equivalent to $g{ }_{\Delta}(Q \cup I) \models A$, where $\models$ is propositional consequence and $\Delta$ is the set of constants in $I$. The problem there is an $I \in \operatorname{rep}(\mathbf{T})$ such that $\operatorname{gr}_{\Delta}(Q \cup I) \not \models A$ can be decided as follows: (1) guess a valuation $\sigma$ for the variables in $\mathbf{T}$ and a propositional valuation $\gamma$ for the atoms in $\operatorname{gr}_{\Delta}(Q \cup \sigma(\mathbf{T}))$ and (2) check $\sigma(\mathbf{T}) \in \operatorname{rep}(\mathbf{T}), \gamma \models g r_{\Delta}(Q \cup \sigma(\mathbf{T}))$, and $\gamma \not \models A$. Clearly, the algorithm runs in NP in the size of $\mathbf{T}$ and in NExp in the combined size. It follows that cert ${ }^{Q}$ can be decided in coNP and cert in coNExp.

We observe that poss can be straightforwardly reduced to cons, and vice versa.

Proposition 7. There exists an LSpace reduction from cons (resp., cons $\left.{ }^{Q}\right)$ for a class of queries $X$ to poss (resp., poss ${ }^{Q^{\prime}}$ ) for $X$, and vice versa.

Therefore, our results for consistency correspond with those for possible answers. When considering C-databases without conditions (called V-databases in [14]), Abiteboul et al. [3] showed that $\operatorname{cert}^{Q}$ is in PTime for Datalog, while poss ${ }^{Q}$ is NP-complete for $\mathrm{nr}$-Datalog queries and cert $^{Q}$ is coNP-complete for nr-Datalog $\urcorner$ queries. We complement these results as follows.

Proposition 8. When considering C-databases without conditions, cert ${ }^{Q}$ is in LSpace for $\mathrm{nr}$-Datalog queries, cert is Exp-complete for Datalog queries, and poss is NExpcomplete for Datalog queries.

Proof. For deciding cert, variables in C-databases without conditions can be treated as constants (Skolemization), and so the database can be treated as if it were a complete database (implicit in [14,24]). Exp-completeness of cert for Datalog and membership in LSpace of cert ${ }^{Q}$ for $\mathrm{nr}$-Datalog queries follows from the results for complete databases.

By Proposition 6, poss is in NExp. Hardness is proved by a slight modification of the proof of Proposition 4: the guess of the next computation step $i$ at time point $j$ is 


\begin{tabular}{|c|c|c|c|c|c|c|}
\hline & cons $^{Q}$ & $\mid$ poss $^{Q} \mid$ & $\operatorname{cert}^{Q}$ & cons & poss & cert \\
\hline nr-Datalog & NP & NP & coNP/LSpace & PSpace & PSpace & PSpace \\
\hline nr-Datalog $\urcorner$ & NP & NP & coNP & PSpace & PSpace & PSpace \\
\hline Datalog & NP & $\mathrm{NP}$ & $\mathrm{coNP} / \mathrm{P}$ & NExp & NExp & coNExp/Exp \\
\hline st-Datalog $\urcorner$ & $\mathbf{N P}$ & NP & coNP & NExp & NExp & coNExp \\
\hline Datalog $\urcorner$ & NP & NP & coNP & NExp & NExp & coNExp \\
\hline$\overline{\text { Datalog }}^{\vee}$ & $\Sigma_{2}^{\mathrm{p}}$ & $\Sigma_{2}^{\mathrm{p}}$ & coNP & NExp $^{\text {NP }}$ & $\operatorname{NExp}^{\text {NP }}$ & coNExp \\
\hline st-Datalog $\urcorner, V$ & $\Sigma_{2}^{\mathrm{p}}$ & $\Sigma_{2}^{\mathrm{p}}$ & $\Pi_{2}^{\mathrm{p}}$ & $\operatorname{NExp}^{\text {NP }}$ & $\operatorname{NExp}^{\text {NP }}$ & $\operatorname{coNExp}^{\mathrm{NP}}$ \\
\hline$\overline{\text { Datalog }\urcorner, \nabla}$ & $\Sigma_{2}^{\mathrm{P}}$ & $\Sigma_{2}^{\mathrm{p}}$ & $\Pi_{2}^{\mathrm{p}}$ & $\operatorname{NExp}^{\mathbf{N P}}$ & $\operatorname{NExp}^{\mathbf{N P}}$ & $\operatorname{coNExp}^{\mathrm{NP}}$ \\
\hline
\end{tabular}

Table 1. Complexity results for $\mathrm{C}$-databases with/without conditions under closedworld interpretation

performed using a single variable $x_{j}$, which may or may not be valuated with a valid $i$. This means that not all $I \in Q(\operatorname{rep}(\mathbf{T}))$ correspond to runs, but still $T$ has an accepting run iff there is an $I \in Q(\operatorname{rep}(\mathbf{T}))$ such that accept $(1) \in I$.

The further complexity results for V-databases are the same as for C-databases. We note that the stated complexity results about V-databases apply even if variables may not occur twice in the database. Such V-databases are called Codd databases in [14].

Table 1 summarizes the complexity results for consistency and query answering under closed-world interpretation (CWI), both for databases with and without conditions (separated by the ' $\%$ symbol). Where the two cases correspond, only one complexity class is written. The results in boldface are novel. Note that all results in the table, save the LSpace result, are completeness results.

We can observe from the table that problems for query languages that are complete for (the complement of) a nondeterministic complexity class when considering complete databases (e.g., Datalog $\urcorner$ ) do not increase in complexity when considering incomplete databases. So, for Datalog with disjunction and/or negation, answering queries on incomplete databases is not harder than answering queries on complete databases.

All considered PTime query languages jump to NP (resp., coNP) when considering data complexity and queries on C-databases. However, differences arise when considering the size of the query: for example, the combined complexity of the Datalog NExp-complete, whereas it is PSpace-complete for nr-Datalog.

Finally, we can observe that problems for queries on databases without conditions are only easier than those with conditions when considering PTime queries without negation and even then only certain answers are easier; possible answers and consistency are just as hard.

\subsection{Complexity of Open-World Interpretation}

For positive queries, certain answers under open-world interpretation (OWI) correspond to certain answers under CWI, which is a straightforward consequence of Lemma 1.

Proposition 9. Let $\mathbf{T}$ be $C$-database, $Q$ a Datalog ${ }^{\vee}$ query, and $A$ a set of facts. Then, $\operatorname{Cert}(A, \mathbf{T}, Q)$ iff $\operatorname{cert}(A, \mathbf{T}, Q)$. 


\begin{tabular}{|c|c|c|c|c|}
\hline & Cons $^{Q}$ & Cert $^{Q}$ & Cons & Cert \\
\hline nr-Datalog & $\mathbf{N P} /$ constant & coNP/LSpace & NP/LSpace & PSpace \\
\hline nr-Datalog $\urcorner$ & Undec. & Undec. & Undec. & Undec. \\
\hline Datalog & NP/constant & coNP/P & $\mathbf{N P} / \mathbf{P}$ & coNExp/Exp \\
\hline st-Datalog ${ }^{7}$ & Undec. & Undec. & Undec. & Undec. \\
\hline Datalog $\urcorner$ & Undec. & Undec. & Undec. & Undec. \\
\hline Datalog $^{\nabla}$ & $\mathbf{N P} /$ constant & coNP & $\Sigma_{2}^{\mathrm{P}}$ & coNExp \\
\hline st-Datalog ${ }^{\neg, \vee}$ & Undec. & Undec. & Undec. & Undec. \\
\hline Datalog $^{\urcorner, V}$ & Undec. & Undec. & Undec. & Undec. \\
\hline
\end{tabular}

Table 2. Complexity results for C-databases with/without conditions under open-world interpretation

Checking $\operatorname{Cons}(\mathbf{T}, Q)$ for consistent $\mathbf{T}$ (i.e., $\operatorname{Rep}(\mathbf{T}) \neq \emptyset$ ) corresponds to checking satisfiability of $Q$, which is known to be decidable for $Q \in$ Datalog [2, Theorem 12.5.2]. Observe that databases without conditions are trivially consistent. We establish the complexity of Cons ${ }^{Q}$ and Cons in the following two propositions.

Proposition 10. Satisfiability of Datalog queries is PTime-hard and satisfiability of Datalog $\vee^{\vee}$ queries is $\Sigma_{2}^{p}$-hard.

Proposition 11. The problems Cons ${ }^{Q}$ and Cons are NP-complete for $\mathrm{nr}$-Datalog and Datalog; Cons ${ }^{Q}$ is NP-complete and Cons is in $\Sigma_{2}^{p}$ for Datalog $\vee$ queries.

When considering C-databases without conditions, Cons is in LSpace for nr-Datalog queries and in PTime for Datalog queries.

Adding negation to any of the considered query languages results in undecidability, by the undecidability of finite satisfiability of nr-Datalog $\urcorner$ queries [22]. Table 2 summarizes the complexity results under OWI where "Undec." is short for "Undecidable" and "constant" means "decidable in constant time". All results, save the two LSpace results, are completeness results.

As can be seen from the table, checking consistency under OWI is often easier than checking consistency under CWI. Intuitively, this is the case because under CWI one needs to take the absence of tuples in the database into account.

\section{Related Work}

Variations on Query Languages Reiter [18] devised an algorithm for evaluating certain answers to queries on logical databases, which are essentially condition-free Cdatabases under CWI. The algorithm, based on relational algebra, is complete for positive first-order queries (i.e., nr-Datalog) and for conjunctive queries extended with negation in front of atomic formulas (i.e., a subsef of st-Datalog $\urcorner$ ). We obtain sound and complete reasoning for free by our translation of queries on $\mathrm{C}$-databases to calculating the stable models of a logic program.

Rosati [19] considers condition- and variable-free databases under OWI and certain answers for conjunctive queries and unions of conjunctive queries, as well as extensions with inequality and negation. The data complexity of such queries is polynomial 
as long as the queries are safe, but becomes undecidable when considering unions of conjunctive queries extended with negation involving universally quantified variables.

We considered nr-Datalog, which generalize (unions of) conjunctive queries, but did not (yet) consider extensions with inequality and restricted forms of negation. A topic for future work is query answering on C-databases for such languages, both under CWI and OWI.

Logic Programming with Open Domains One traditionally assumes in Logic Programming information regarding individuals is complete. Hence, the grounding of logic programs with the constants in the program. Approaches that allow for incomplete information in that sense, e.g., where one does not need all relevant constants in the program to deduce correct satisfiability results, are, the finite $k$-belief sets of [10,20,21] and its generalization $^{3}$, open answer sets [13]. Both deal with incomplete information by not a priori assuming that all relevant constants are present in the program under consideration. It is not clear what the exact relation with $\mathrm{C}$-databases is; this is part of future work.

Or-sets An alternative way of representing incomplete information is through objects with or-sets [15]. For example, a tuple (John, $\{30,31\})$ indicates that John has age 30 or 31 . This notion of incompleteness (which assumes closed-world interpretation) is somewhat simpler than C-databases and could be simulated using disjunctions of equality atoms. In [15], one shows that certain answers for existentially quantified conjunctive first-order formulas are data complete for coNP. A result that conforms with the coNP result for $\operatorname{cert}^{Q}$ with nr-Datalog queries in Table 1.

\section{Outlook}

We studied query languages ranging from nr-Datalog to Datalog $\neg, \vee$. Besides extensions of positive query languages (including conjunctive queries) with inequality and limited forms of negation (e.g., only in front of extensional predicates), in future work we plan to consider integrity constraints, both as part of the query language, as is common in logic programming, and as part of the database. While under OWI adding integrity constraints to the database leads to undecidability already for very simple query languages [19], query answering under integrity constraints for databases under CWI is largely uncharted territory. We suspect that there are cases that are undecidable under OWI, but solvable under CWI, because by Lemma 2 we need to consider only a finite subset of $\operatorname{rep}(\mathbf{T})$. We note that Vardi [24] showed that checking integrity of an incomplete database is often harder under CWI than under OWI.

Abiteboul and Duschka [1] argue that a materialized view (e.g., the result of data integration) should be seen as an incomplete database, where the source predicates are seen as incomplete. Indeed, viewing a global schema as a sound view - essentially a condition- and variable-free incomplete database - is common in data integration $[12,16]$. Considering variables and, possibly, also conditions in (materialized) global views is a natural extension in this scenario; for example, local relations may have fewer columns than global relations, requiring view definitions of the form $\exists Y . v(X, Y, Z) \leftarrow$ $s(X, Z)$. In future work we intend to consider query answering using such views.

\footnotetext{
${ }^{3}$ Both finite and infinite open answer sets are allowed.
} 
Acknowledgements We thank the anonymous reviewers for useful comments and feedback. The work in this paper was partially supported by the European Commission under the project ONTORULE (IST-2009-231875).

\section{References}

1. S. Abiteboul and O. M. Duschka. Complexity of answering queries using materialized views. In Proc. PODS, pp. 254-263, 1998.

2. S. Abiteboul, R. Hull, and V. Vianu. Foundations of Databases. Addison-Wesley, 1995.

3. S. Abiteboul, P. C. Kanellakis, and G. Grahne. On the representation and querying of sets of possible worlds. Theoretical Computer Science, 78(1):158-187, 1991.

4. L. E. Bertossi and L. Bravo. Consistent query answers in virtual data integration systems. In Inconsistency Tolerance, LNCS 3300:42-83, 2005.

5. E. F. Codd. Extending the database relational model to capture more meaning. ACM ToDS, 4(4):397-434, 1979.

6. E. Dantsin, T. Eiter, G. Gottlob, and A. Voronkov. Complexity and expressive power of logic programming. ACM Computing Surveys, 33(3):374-425, 2001.

7. T. Eiter, G. Gottlob, and H. Mannila. Disjunctive Datalog. ACM ToDS, 22(3):364-418, 1997.

8. R. Fagin, P. G. Kolaitis, R. J. Miller, and L. Popa. Data exchange: semantics and query answering. Theor. Comput. Sci., 336(1):89-124, 2005.

9. M. Gelfond and V. Lifschitz. Classical negation in logic programs and disjunctive databases. New Generation Computing, 9(3-4):365-386, 1991.

10. M. Gelfond and H. Przymusinska. Reasoning on open domains. In Proc. LPNMR, pp. $397-$ $413,1993$.

11. G. Grahne. Horn tables - an efficient tool for handling incomplete information in databases. In Proc. PODS, pp. 75-82, 1989.

12. A. Y. Halevy. Answering queries using views: A survey. VLDB J., 10(4):270-294, 2001.

13. S. Heymans, D. V. Nieuwenborgh, and D. Vermeir. Open answer set programming with guarded programs. ACM ToCL, 9(4), 2008.

14. T. Imieliński and W. Lipski. Incomplete information in relational databases. Journal of the ACM, 31(4):761-791, 1984.

15. T. Imieliński, S. A. Naqvi, and K. V. Vadaparty. Incomplete objects - a data model for design and planning applications. In Proc. SIGMOD, pp. 288-297, 1991.

16. M. Lenzerini. Data integration: A theoretical perspective. In PODS, pp. 233-246, 2002.

17. L. Libkin. Data exchange and incomplete information. In Proc. PODS, pp. 60-69, 2006.

18. R. Reiter. A sound and sometimes complete query evaluation algorithm for relational databases with null values. Journal of the ACM, 33(2):349-370, 1986.

19. R. Rosati. On the decidability and finite controllability of query processing in databases with incomplete information. In Proc. PODS, pp. 356-365, 2006.

20. J. Schlipf. Some Remarks on Computability and Open Domain Semantics. In Proc. WS on Structural Complexity and Recursion-Theoretic Methods in Logic Programming, 1993.

21. J. Schlipf. Complexity and Undecidability Results for Logic Programming. Annals of Mathematics and Artificial Intelligence, 15(3-4):257-288, 1995.

22. B. Trakhtenbrot. Impossibility of an algorithm for the decision problem for finite models. Dokl. Akad. Nauk SSSR, 70:596-572, 1950.

23. M. Y. Vardi. The complexity of relational query languages (extended abstract). In $A C M$ Symposium on Theory of Computing, pp. 137-146, 1982.

24. M. Y. Vardi. On the integrity of databases with incomplete information. In Proc. PODS, pp. 252-266, 1986.

25. M. Y. Vardi. Querying logical databases. Journal of Computer and System Sciences, 33(2):142-160, 1986.

26. S. G. Vorobyov and A. Voronkov. Complexity of nonrecursive logic programs with complex values. In PODS, pp. 244-253, 1998. 